Article

\title{
Evaluating Serum Heat Shock Protein Levels as Novel Biomarkers for Atrial Fibrillation
}

\author{
Denise M. S. van Marion ${ }^{1,+}$, Eva A. H. Lanters ${ }^{2,+}$, Kennedy S. $\operatorname{Ramos}^{1,2}{ }^{\mathbb{D}}$, Jin Li ${ }^{1}$, \\ Marit Wiersma 1,3, Luciënne Baks-te Bulte ${ }^{1}$, Agnes J. Q. M. Muskens ${ }^{2}$, Eric Boersma ${ }^{2}$, \\ Natasja M. S. de Groot ${ }^{2, \ddagger(\mathbb{D})}$ and Bianca J. J. M. Brundel ${ }^{1, *, \ddagger \mathbb{D}}$ \\ 1 Department of Physiology, Amsterdam Cardiovascular Sciences, Amsterdam UMC, Vrije University, \\ 1081HV Amsterdam, The Netherlands; d.vanmarion@amsterdamumc.nl (D.M.S.v.M.); \\ k.silvaramos@amsterdamumc.nl (K.S.R.); j.li@amsterdamumc.nl (J.L.); \\ m.wiersma1@amsterdamumc.nl (M.W.); lucienne.baks@gmail.com (L.B.-t.B.) \\ 2 Department of Cardiology, Erasmus MC, 3000CA Rotterdam, The Netherlands; \\ Eva_Lanters@hotmail.com (E.A.H.L.); a.muskens@erasmusmc.nl (A.J.Q.M.M.); \\ h.boersma@erasmusmc.nl (E.B.); n.m.s.degroot@erasmusmc.nl (N.M.S.d.G.) \\ 3 Netherlands Heart Institute, 3511EP Utrecht, The Netherlands \\ * Correspondence: b.brundel@amsterdamumc.nl; Tel.: +31-6-2733-9910 \\ + These authors contributed equally to this paper. \\ $\ddagger$ These authors contributed equally to this paper.
}

Received: 23 July 2020; Accepted: 14 September 2020; Published: 16 September 2020

Abstract: Background: Staging of atrial fibrillation (AF) is essential to understanding disease progression and the accompanied increase in therapy failure. Blood-based heat shock protein (HSP) levels may enable staging of AF and the identification of patients with higher risk for AF recurrence after treatment. Objective: This study evaluates the relationship between serum HSP levels, presence of AF, AF stage and AF recurrence following electrocardioversion (ECV) or pulmonary vein isolation (PVI). Methods: To determine HSP27, HSP70, cardiovascular (cv)HSP and HSP60 levels, serum samples were collected from control patients without AF and patients with paroxysmal atrial fibrillation (PAF), persistent (PeAF) and longstanding persistent (LSPeAF) AF, presenting for ECV or PVI, prior to intervention and at 3-, 6- and 12-months post-PVI. Results: The study population $(n=297)$ consisted of 98 control and 199 AF patients admitted for ECV $(n=98)$ or PVI $(n=101)$. HSP27, HSP70, cvHSP and HSP60 serum levels did not differ between patients without or with PAF, PeAF or LSPeAF. Additionally, baseline HSP levels did not correlate with AF recurrence after ECV or PVI. However, in AF patients with AF recurrence, HSP27 levels were significantly elevated post-PVI relative to baseline, compared to patients without recurrence. Conclusions: No association was observed between baseline HSP levels and the presence of AF, AF stage or AF recurrence. However, HSP27 levels were increased in serum samples of patients with AF recurrence within one year after PVI, suggesting that HSP27 levels may predict recurrence of AF after ablative therapy.

Keywords: atrial fibrillation; heat shock protein; electrical cardioversion; pulmonary vein isolation; biomarkers

\section{Introduction}

Atrial fibrillation (AF) is the most common cardiac arrhythmia, with a rising prevalence due to the aging population [1]. Proper staging of AF is essential to select the optimal treatment strategies to prevent AF progression, and the accompanied risk to develop severe complications such as thromboembolic events, heart failure, cognitive impairment and increased mortality [2,3]. At present, AF can only be diagnosed with a surface electrocardiogram when a patient already suffers from AF, 
presenting with palpitations or thromboembolic complications. However, diagnosis of AF may be challenging in patients with asymptomatic or very short-lasting episodes of AF [4]. In addition, treatment aimed at rhythm control, such as ablative therapy, is less successful in patients with persistent AF, compared to paroxysmal AF [3]. Hence, proper staging of AF and the start of effective treatment of AF is of utmost importance. Therefore, there is an urgent need to identify diagnostic biomarkers to stage AF and guide patient-tailored therapy [5].

Biomarkers are widely accepted as a diagnostic tool to screen or monitor patients for a variety of cardiovascular diseases. At present, there are no recommendations on the use of AF-specific biomarkers in the most recent guidelines [3,6], despite the fact that several blood-based biomarkers related to AF pathology have been identified. These biomarkers include brain natriuretic peptides, cancer-antigen-125, fibroblast growth factor-23 [7,8] and -21 [9], highly sensitive cardiac troponin I [10], homocysteine [11], (a)symmetric dimetylarginine [12], interleukine-6 and matrix metalloproteinase-9/tissue inhibitor of metalloproteinase-1 ratio [13]. Although potential AF-related biomarkers are available, the role of these biomarkers in staging of AF (paroxysmal or (longstanding) persistent AF) or predicting AF recurrence after AF therapy has only be moderately studied.

Emerging evidence indicates that heat shock proteins (HSPs) may represent a suitable biomarker to predict AF recurrence after treatment. HSPs are chaperones that play an important role in safeguarding proteostasis, the homeostasis of protein expression, and function and degradation in cells [14]. The derailment of proteostasis has been identified as a key factor underlying electropathology and AF progression [14-16]. During stress or disease, such as AF, especially, activation of the heat shock transcription factor 1 regulates HSP transcription [17]. Within the HSP family, small HSPs, including HSP27, are probably the most important in maintaining proteostasis in cardiomyocytes by stabilizing the contractile proteins [18-21]. Previously, atrial HSP27 levels were found to be induced in atrial tissue samples of patients with paroxysmal AF, while tissue HSP27 levels get exhausted in patients with (longstanding) persistent AF [18], indicating that low tissue HSP levels are associated with AF progression. The study of Hu et al. described that low baseline serum HSP27 levels of patients who received ablative therapy predict AF recurrence and patients with high baseline serum levels of HSP27 showed an improved maintenance rate of sinus rhythm [22]. So far, it is unknown which HSP family member(s) represent biomarkers to identify the stage of AF and recurrence after therapy. In the current study, various members of the HSP family, including HSP27, HSP70, cardiovascular (cv)HSP and HSP60, were measured in serum samples of control and patients with paroxysmal atrial fibrillation (PAF), persistent atrial fibrillation (PeAF) and longstanding persistent atrial fibrillation (LSPeAF), undergoing elective electrical cardioversion (ECV) or pulmonary vein isolation (PVI), to identify whether HSPs associate with the stage of AF and recurrences after either PVI or ECV. Herein, we report that baseline HSP levels between control and AF patients are comparable. However, HSP27 levels were increased in follow-up samples of patients with AF recurrence after PVI, suggesting that increased HSP27 levels may predict recurrence of AF after ablative therapy.

\section{Materials and Methods}

\subsection{Study Popultion}

From December 2014 till November 2016, 297 subjects $>18$ years with or without a history of AF were prospectively enrolled for the HALT \& REVERSE study [23] at the department of cardiology in the Erasmus MC, Rotterdam, the Netherlands. The study population consists of a control group of patients without a history of AF and a study group of patients with symptomatic AF who were scheduled for electrical cardioversion (ECV) or pulmonary vein isolation (PVI).

The control group consisted of patients $(n=98)$ who were scheduled for elective ablation of premature ventricular contractions (PVC), Wolff-Parkinson-White syndrome (WPW) or Ajmaline testing. These patients were eligible for inclusion in case of absence of structural heart disease and any 
atrial tachyarrhythmia. Blood serum samples were obtained 1 day prior to the scheduled intervention. Follow-up was not performed.

The study group included patients presenting for ECV $(n=98)$ or PVI $(n=101)$ for either symptomatic PAF (less than 7 days of AF), PeAF (having AF between 7 days and 1 year) or LSPeAF (more than 1 year of AF). Exclusion criteria included paced atrial rhythms, cancer, inflammation and rheumatic diseases. Blood samples were obtained 1 day prior to the scheduled intervention. PVI was unsuccessful in two patients.

Patients who underwent PVI visited the outpatient clinic at 3-, 6- and 12-months after the procedure to provide follow-up serum samples and to screen for AF recurrences. Due to rescheduling of patients to different hospitals, follow-up serum samples were not available for some patients in both the AF recurrence and no recurrence group. In addition, AF recurrence data is available via recordings of inbetween visits due to AF complaints of the patients. AF recurrence was defined as an AF episode documented on either a 12-lead surface ECG or Holter monitor recordings. In patients undergoing ECV, for each individual patient only 3-, 6- and 12-months post-procedural follow-up telephone consultations were scheduled. The study endpoint was the completion of the 12-months follow-up period or earlier due to withdrawn informed consent, pacemaker implant or AF recurrence. Clinical characteristics were obtained from the electronic patients' files.

All patients signed written informed consent prior to inclusion. This sub-study is part of the HALT AND REVERSE trial (MEC-2014-393), and is approved by the institutional medical ethical committee. The study is carried out according to the principals of the Declaration of Helsinki in accordance with the Medical Research Committee involving the Human Subjects Act.

\subsection{HSP Measurement in Serum Samples}

Immediately after blood sample collection, serum was harvested from blood in BD Vacutainer ${ }^{\mathrm{TM}} \mathrm{SST}^{\mathrm{TM}}$ II Advance Tubes (Fisher Scientific, Bleiswijk, the Netherlands) by centrifugation at $2000 \times g$ for $10 \mathrm{~min}$ at $4{ }^{\circ} \mathrm{C}$ and frozen in $-80^{\circ} \mathrm{C}$ until analysis of HSP27, HSP70, cvHSP and HSP60 levels. For measurement of serum HSP27 levels, samples were diluted six times, and for HSP70 levels samples were diluted twice in 1\% BSA in PBS. The amount of HSP27 and HSP70 protein was detected in triplicates using human HSP27 or HSP70 DuoSet ${ }^{\circledR}$ ELISA kits from R\&D (Cat. no. DY1580 and DY1663, respectively, Minneapolis, MN, USA) according to the manufacturer's instructions with minor adjustments (serum was incubated at $4{ }^{\circ} \mathrm{C}$ overnight, instead of $2 \mathrm{~h}$ at room temperature). Undiluted serum was used to measure cvHSP protein (singular) with ELISA kits from Cusabio (CSB-EL010838HU, Houston, TX, USA), according to manufacturer's instructions with minor adjustments (kept incubation temperature at $20^{\circ} \mathrm{C}$ ). Undiluted serum was used to measure HSP60 protein in duplicate with the HSP60 DuoSet ${ }^{\circledR}$ ELISA kit from R\&D (DYC1800), according to manufacturer's instructions.

\subsection{Statistical Analysis}

Data were analyzed with SPSS Statistics version 26.0 for Windows (SPSS, Inc., Chicago, IL, USA) and GraphPad Prism version 8.0 (Graphpad Software Inc., San Diego, CA, USA). All data were tested for Gaussian distribution. Continuous and normally distributed data are presented as mean \pm standard deviation (SD), non-normally distributed data as median [interquartile range (IQR)], and categorical data as number (percentage). Differences in clinical characteristics and HSP levels between patients with and without AF were tested with independent-samples $t$-test, Mann-Whitney and Chi-square test. Differences in clinical characteristics and HSP levels between patients without a history of AF, PAF, PeAF and LSPeAF were tested with one-way analysis of variance (ANOVA), Kruskal-Wallis test, Chi-square test and Fisher's Exact test. When serum HSP levels were below detection limit of the ELISA (only for $n=7 \mathrm{cvHSP}$ and $n=41 \mathrm{HSP} 60$ samples), values at the lower limit of detection were used for statistical analysis. HSP levels are not normally distributed and are Log-transformed for statistical analysis (original HSP values are presented in Tables and Figures). Uni- and multivariate linear regression was used to correlate baseline serum HSP levels with clinical parameters, and bivariate spearman 
correlation was used to correlate AF recurrence with clinical parameters. The difference between baseline and follow-up serum HSP levels was calculated with a repeated measures model. To relate HSP levels over time with AF recurrence and to analyze the sensitivity of the results, sensitivity analysis was performed using joint modeling. Hereto, the occurrence of the first AF recurrence (endpoint) in relation to the standardized (' $\mathrm{Z}$ ') value of $\log 2(\mathrm{HSP})$ was modeled, while using all measurements up to and including the moment of the first AF recurrence in endpoint event cases, and all measurements in those who remained event-free. A two-sided $p$ value of $<0.05$ indicates statistical significance.

\section{Results}

\subsection{Study Population}

The entire study population consisted of 297 patients (67\% males, age $56.7 \pm 13.4$ years), including a control group of 98 patients without AF and a study group of 199 patients with either PAF $(n=86$, $29 \%)$, PeAF $(n=108,36.4 \%)$ or LSPeAF $(n=5,1.7 \%)$ AF. Table 1 outlines baseline characteristics of the entire study population. Patients with AF were older $(p<0.001)$, more often male $(p<0.001)$, had a higher BMI $(p<0.001)$, more often had hypertension $(p<0.001)$, more often had diabetes mellitus $(p<0.001)$ and more often had dyslipidemia $(p<0.05)$, compared to patients without AF (Table 1$)$. Clinical parameters did not differ between patients with paroxysmal and persistent AF, except for an impaired LVF $(p<0.001)$ and larger left atrial volume $(p<0.001)$ in the persistent AF patients.

Table 1. Clinical characteristics of the study population.

\begin{tabular}{|c|c|c|c|c|c|}
\hline & Control & PAF & PeAF & LSPeAF & All AF Patients \\
\hline \multicolumn{6}{|l|}{ Group, $n(\%)$} \\
\hline Electro cardioversion (ECV) & - & $12(14)$ & $83(76.9)$ & $3(60)$ & $98(49)$ \\
\hline Pulmonary vein isolation (PVI) & - & $74(86)$ & $25(23.1)$ & $2(40)$ & $101(51)$ \\
\hline Age (years), mean \pm SD & $48.2 \pm 15.3$ & $61.3 \pm 9.5^{* * *}$ & $60.8 \pm 10.6^{* * *}$ & $57.5 \pm 9$ & $60.9 \pm 10.1^{* * *}$ \\
\hline Hypertension, yes, $n(\%)$ & $23(23.5)$ & $43(50)^{* * *}$ & $51(47.2) * *$ & $3(60)$ & $97(48.7)^{* * *}$ \\
\hline Diabetes mellitus, yes, $n(\%)$ & $5(5.1)$ & $10(11.6)$ & $15(13.9)$ & $1(20)$ & $26(13.1) *$ \\
\hline Dyslipidemia, yes, $n(\%)$ & $16(16.3)$ & $25(29.1)$ & $33(30.6)$ & $3(60)$ & $61(30.7)^{* *}$ \\
\hline Thyroid disease, yes, $n(\%)$ & $2(2)$ & $4(4.7)$ & $8(7.4)$ & $1(20)$ & $13(6.5)$ \\
\hline Left ventricular function (LVF), $n(\%)$ & & & $* \# \#$ & & \\
\hline Missing $^{\dagger}$ & 21 & 0 & 5 & 0 & 5 \\
\hline Left atrial volume index & 27.9 & 38.6 & 47 & 43.1 & 41.1 \\
\hline$\left(\mathrm{mL} / \mathrm{m}^{2}\right)$, median [IQR] & {$[21.2-39.7]$} & {$[29.3-48.4] *$} & {$[35.7-60.5] * * \# \#$} & [25.9-73.6] & {$[31.8-54]^{* *}$} \\
\hline \multicolumn{6}{|l|}{ Drugs, yes, $n(\%)$} \\
\hline Drugs total & $52(53.6)$ & $84(97.7) * * *$ & $104(96.3) * * *$ & $5(100)$ & $193(97)^{* * *}$ \\
\hline ACE. ARB. AT2 antagonist & $26(26.8)$ & $40(46.5) *$ & $48(44.9)^{*}$ & $3(60)$ & $91(46)^{* *}$ \\
\hline Statin & $17(17.5)$ & $32(37.2) *$ & $37(34.3) *$ & $4(80) *$ & $73(36.7) * * *$ \\
\hline Antiarrhythmic drugs (AAD) total $\ddagger$ & $43(44.3)$ & $79(91.9)^{* * *}$ & $103(95.4)^{* * *}$ & $5(100)$ & $187(94)^{* * *}$ \\
\hline Class I AAD & $5(5.2)$ & $31(36.0)^{* * *}$ & 14 (13) \#\#\# & $1(20)$ & $46(23.1)^{* * *}$ \\
\hline Class II AAD & $31(32)$ & $36(41.9)$ & $55(50.9)$ * & $1(20)$ & $92(46.2)^{*}$ \\
\hline Class III AAD & $6(6.2)$ & $42(48.8)$ & 55 (50.9) & $2(40)$ & $99(49.7)^{* * *}$ \\
\hline
\end{tabular}

${ }^{\dagger}$ The percentages of LVF are valid percentages and corrected for the missing values; ${ }^{\ddagger}$ Patients may use more than one type of AAD; therefore, the sum of all classes is not equal to total; ${ }^{*} p<0.05,{ }^{* *} p<0.01$ and ${ }^{* * *} p<0.001$ compared to control ${ }^{\# \#} p<0.01$ and ${ }^{\# \#} p<0.001$ comparing paroxysmal AF with persistent AF.

\subsection{Baseline HSP Levels Related to Clinical Stage of AF}

To study the relationship between baseline HSP levels and the stage of AF, HSP27, HSP70, cvHSP and HSP60 levels were determined in serum samples of PAF, PeAF and LSPeAF patients and 
compared to controls. Figure 1 shows baseline concentrations of serum HSP27, HSP70, cvHSP and HSP60 of both control and AF patients; corresponding values are depicted in Supplemental Table S1. These findings and the absence of a correlation between AF stage and HSP levels after correction for potential confounders in a multivariate model (Supplemental Tables S2 and S3) indicate that there are no differences in serum HSP values between the control patients and AF patients with PAF, PeAF and LSPeAF.
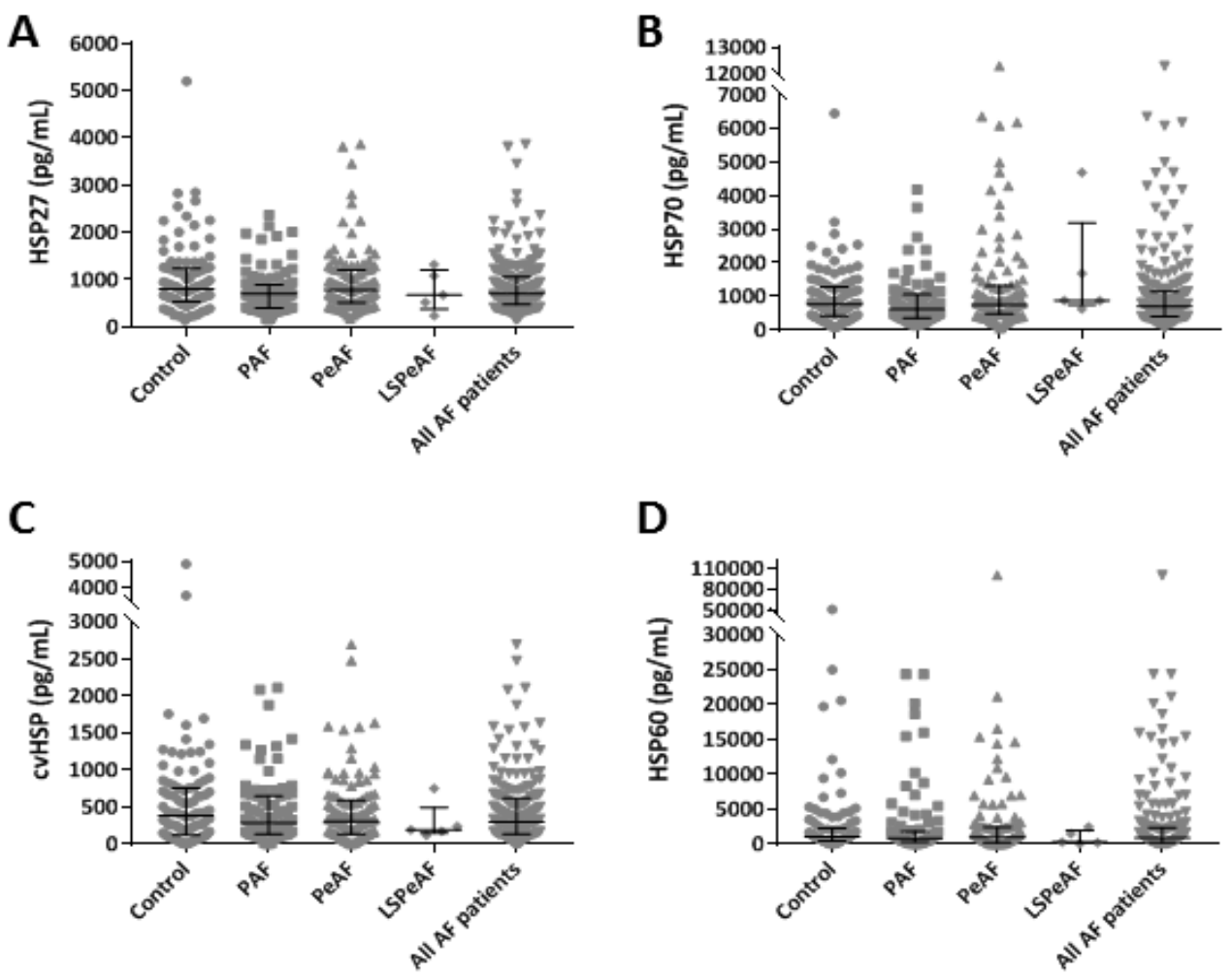

Figure 1. Baseline heat shock protein (HSP) serum levels in patients without and with (paroxysmal, persistent and longstanding persistent) atrial fibrillation (AF). HSP27 (A), HSP70 (B), cvHSP (C) and HSP60 (D) expression levels (pg/mL) in baseline serum of control, paroxysmal atrial fibrillation (PAF), persistent atrial fibrillation (PeAF), longstanding persistent atrial fibrillation (LSPeAF) and all AF patients.

\subsection{Relation between Baseline HSP Levels and AF Recurrence}

In the total AF population, AF recurrence was significantly correlated to AF stage, age, dyslipidemia and LVF, but not related to medication usage (Supplemental Table S4).

After ECV, AF recurrence was determined within 3 months $(n=52,53.1 \%), 6$ months $(n=55$, $56.1 \%)$ and one year $(n=64,65.3 \%)$ (Supplemental Table S5). Figure 2 shows the distribution of the various HSP levels in patients with AF recurrence (red), compared to the remainder of the ECV population (green). Baseline HSP27, HSP70, cvHSP and HSP60 levels did not differ between patients with or without AF recurrences within the first year after ECV (Figure 2, Supplemental Tables S5 and S6).

AF recurrences after PVI occurred within 3 months $(n=34,34 \%), 6$ months $(n=47,47 \%)$ and within 1 year $(n=59,58 \%)$ (Supplemental Table S7). For all time points, AF episodes were more often observed in patients with PeAF or LSPeAF, compared to patients with PAF $(p<0.01)$. Baseline serum HSP levels did not discriminate between patients with and without AF recurrence, as demonstrated in Figure 3 , and Supplemental Table S6. However, HSP27 and HSP70 levels were significantly increased at 3-, 6- and 12-months post-PVI treatment in patients with an AF recurrence within one year, compared to baseline levels (Figure 4 and Supplemental Table S6). The increase in serum HSP27 levels, and not HSP70 
levels, corrected for repeated measures, was significantly associated with AF recurrence $(p<0.013)$, substantiating the role of HSP27 in the prediction of AF recurrence after PVI.
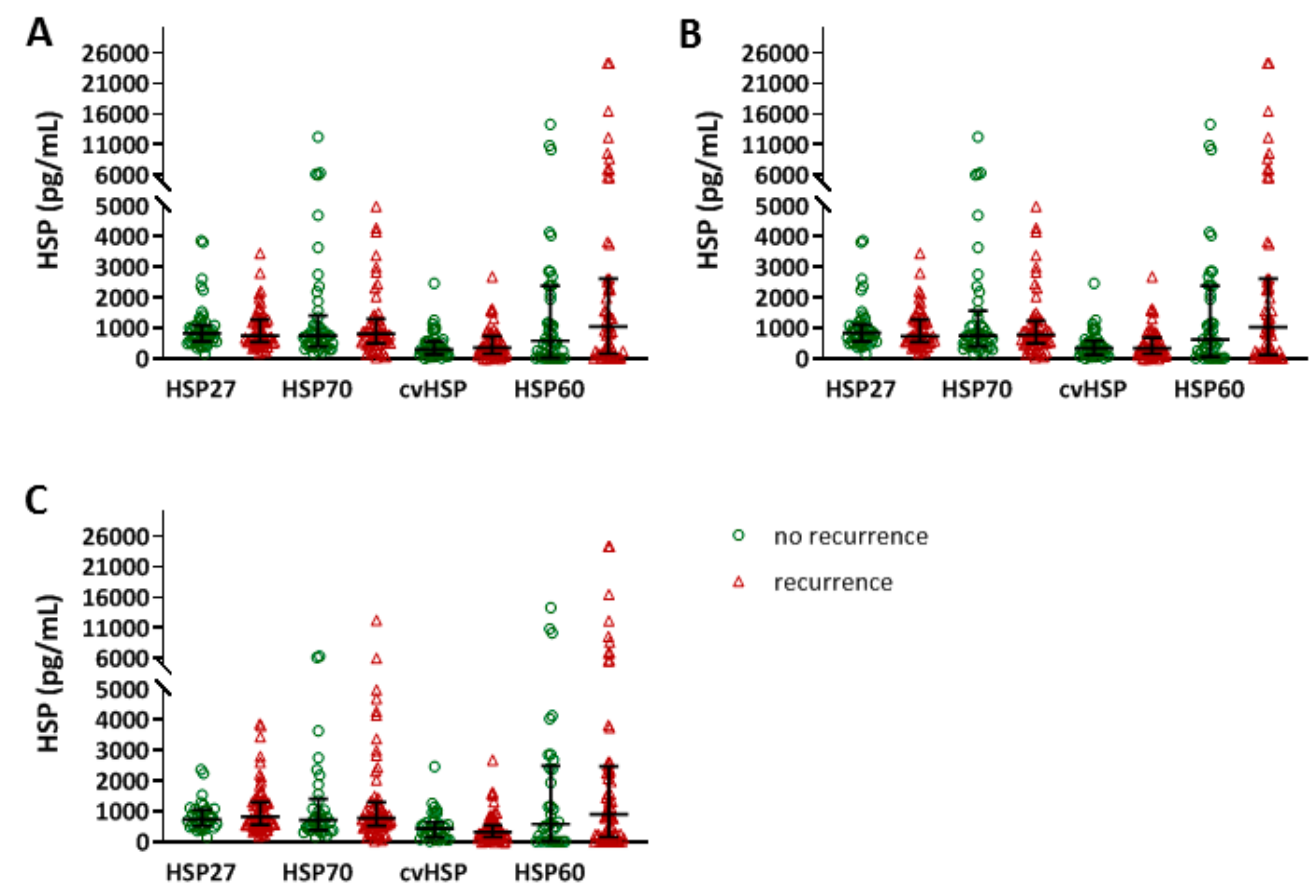

Figure 2. No differences in baseline HSP27, HSP70, cvHSP or HSP60 serum levels between patients with and without AF recurrence after electro cardioversion (ECV). HSP27, HSP70, cvHSP and HSP60 serum levels (pg/mL) at baseline, comparing patients with and without AF recurrence within 3 months (A), 6 months (B) and 1 year (C) after ECV treatment.
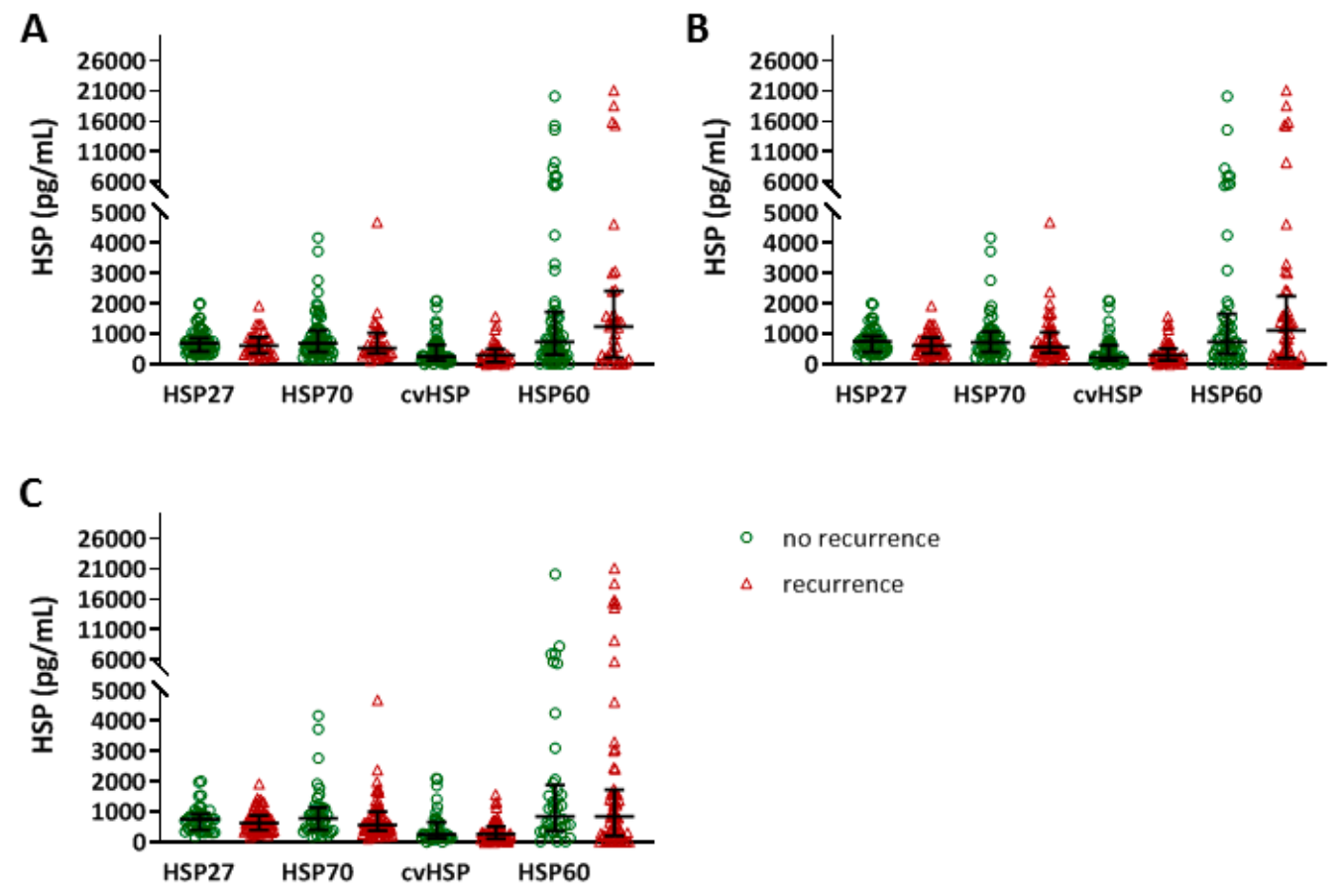

Figure 3. No differences in baseline HSP27, HSP70, cvHSP or HSP60 serum levels between patients with and without AF recurrence after PVI. HSP27, HSP70, cvHSP and HSP60 serum levels (pg/mL) at baseline, comparing patients with and without AF recurrence within 3 months (A), 6 months (B) and 1 year (C) after pulmonary vein isolation (PVI) treatment. 

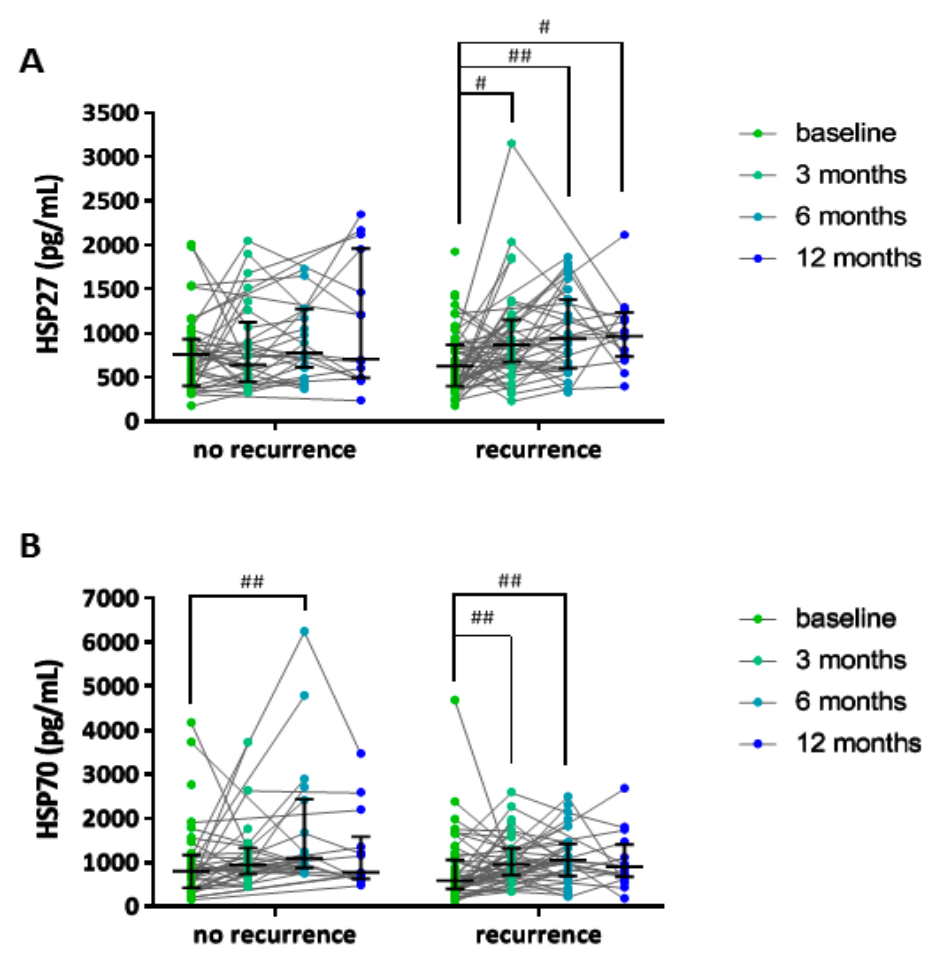

Figure 4. HSP27 and HSP70 levels in follow-up serum were higher than baseline in patients having AF recurrence. HSP27 serum levels $(\mathrm{pg} / \mathrm{mL})$ at baseline and at 3-, 6- and 12-months follow-up in patients having AF recurrence within one year, compared to patients not having AF recurrence (A). HSP70 serum levels (pg/mL) at baseline and at 3-, 6- and 12-months follow-up in patients having AF recurrence within one year, compared to patients not having AF recurrence (B). ${ }^{\#} p<0.05$ and ${ }^{\# \#} p<0.01$ compared to baseline serum HSP levels.

To relate HSP levels over time with AF recurrence as an endpoint, joint modeling was utilized. Therefore, the occurrence of first AF recurrence (endpoint) in relation to the standardized ("Z") value of $\log 2$ (HSP) was modeled, while using all measurements up to and including the moment of the first $\mathrm{AF}$ recurrence in endpoint event cases, and all measurements in those who remained event-free. The results are depicted in the Table 2 (row "all events"). One standard deviation (SD) difference in log2 HSP27 level was associated with a hazard ratio (HR) of 1.32 for having an AF recurrence, supporting the outcomes from the repeated measure model. Unfortunately, the joint modeling for HSP70 did not converge, and therefore no reliable HR estimate could be obtained.

Table 2. Outcomes joint modeling of AF recurrence in relation to HSP levels, and sensitivity analyses.

\begin{tabular}{|c|c|c|c|c|c|c|c|c|c|c|c|c|}
\hline & \multicolumn{2}{|c|}{ Patients } & \multicolumn{2}{|c|}{ Samples } & \multicolumn{4}{|c|}{ HSP27 } & \multicolumn{4}{|c|}{ HSP70 } \\
\hline & AF & AF-Free & $\mathbf{A F}$ & AF-Free & HR & $\begin{array}{c}95 \% \mathrm{CI} \\
\mathrm{LL}\end{array}$ & $\begin{array}{c}95 \% \mathrm{CI} \\
\text { UL }\end{array}$ & $p$-Value & HR & $\begin{array}{c}95 \% \mathrm{CI} \\
\text { LL }\end{array}$ & $\begin{array}{c}95 \% \mathrm{CI} \\
\text { UL }\end{array}$ & $p$-Value \\
\hline $\begin{array}{l}\text { Complete } \\
\text { until } 3 \mathrm{~m}\end{array}$ & 21 & 54 & 42 & 129 & 1.31 & 0.84 & 2.89 & 0.258 & 0.97 & 0.56 & 1.5 & 0.969 \\
\hline $\begin{array}{l}\text { Complete } \\
\text { until } 12 \mathrm{~m}\end{array}$ & 31 & 13 & 70 & 34 & \multicolumn{4}{|c|}{ model does not converge } & \multicolumn{4}{|c|}{ model does not converge } \\
\hline
\end{tabular}

\subsection{Sensitivity Analysis Non-Random Dropout}

Although an association between HSP27 levels and the recurrence of AF after PVI was observed, sensitivity analyses were conducted to evaluate the robustness of our findings in relation to possible 
non-random drop out. Therefore, joint models based on the following sensitivity datasets were run: all available data (all events); patients with an AF recurrence at 3 months who also had an HSP measurement at 3 months (21 cases), in combination with patients who were free of AF recurrence at 3 months and with an HSP measurement $\geq 3$ months (54 patients) (complete until $3 \mathrm{~m}$ ); patients with an AF recurrence at 3 or 6 months who also had an HSP measurement at the moment of the $\mathrm{AF}$ recurrence ( 29 cases), in combination with patients who were free of AF recurrence at 6 months and with an HSP measurement $\geq 6$ months ( 36 patients) (complete until $6 \mathrm{~m}$ ); and patients with an AF recurrence at 3-, 6- or 12-months who also had an HSP measurement at the moment of the AF recurrence (31 cases), in combination with patients who were free of AF recurrence at 12 months and with an HSP measurement at 12 months (13 patients) (complete until $12 \mathrm{~m}$ ). The findings of the sensitivity analyses are provided in Table 2. HSP27 levels were associated with an HR of 2.03 for having an AF recurrence within 6 months post PVI treatment. The results confirm that HSP27 is associated with AF recurrence, and may be used as a predictor. However, HSP70 does not seem to be associated with AF recurrence.

\section{Discussion}

In this study, we observed that serum HSP27, HSP70, cvHSP and HSP60 levels in control patients and patients with paroxysmal and (longstanding) persistent AF were comparable between the groups. Thus, HSP27, HSP70, cvHSP or HSP60 levels could not discriminate between the different AF stages in the total AF population. Additionally, at baseline, HSP27 and HSP70 serum levels were similar in patients without and with AF recurrence within one year after treatment. However, both HSP27 and HSP70 levels were higher at 3-, 6- and 12- months post-PVI compared to baseline levels in patients with AF recurrence within one year. After joint modeling and sensitivity analyses, only increased HSP27 levels post-PVI remained as a predictor of AF recurrence.

\subsection{Heat Shock Proteins Are Not Biomarkers to Differentiate the Stage of Atrial Fibrillation}

There is a great need for biomarkers to stage AF and to improve the selection of the proper treatment for patients. Several (AF-related) serum biomarkers are routinely measured in clinical practice, such as natriuretic peptide, troponin I, troponin T, creatinine and C-reactive protein [24-26], but lack specificity for AF. Our findings indicate no role for HSP27, HSP70, cvHSP or HSP60 as a biomarker for the presence or staging of AF. Our findings are in contrast to findings observed in other studies, as reports revealed a correlation between serum HSP levels and AF. Hu et al. revealed an association between baseline serum HSP27 levels and AF. In this study, serum HSP27 levels were reduced in paroxysmal AF and (longstanding) persistent AF patients, compared to controls in normal sinus rhythm [22]. Additionally, low serum HSP27 was associated with larger left atrial diameter, low atrial voltage (indication of fibrosis [27]) and non-pulmonary ectopies. In addition, serum HSP27 could predict sinus rhythm maintenance and low serum HSP27 correlated with a higher AF recurrence rate [22]. Our study could not confirm these findings. On the contrary, we observed an increase in follow-up serum HSP27 and HSP70 levels in patients who developed AF recurrence post-PVI. In addition, the increased HSP27 levels significantly correlate with AF recurrence. Despite this interesting observation, due to the current study design we cannot determine whether the increase in serum HSP levels is correlated with the duration of the AF episodes and whether this increase started prior or post AF recurrence.

Serum HSP70 levels were similar in control and AF patients in our study and that of others [22,28]. In line with our study, no correlation between clinical or echocardiographic variables and serum HSP70 levels was found and increased serum HSP70 levels at 6 months post-PVI correlated with AF recurrence, substantiating our findings [28].

In patients who underwent CABG, baseline serum HSP70 levels were not predictive for post-operative AF $[29,30]$. While no association between serum HSP60 level and occurrence of AF was found by Maan et al. [31], Cao et al. [32] described that patients undergoing mitral valve 
replacement with AF had higher plasma HSP60 levels than patients in sinus rhythm. Higher plasma HSP60 levels were also predictive for early ( $<7$ days) post-operative AF [32]. Although not the protein itself, pre- and post- operative circulating anti-HSP60 antibodies are associated with post-operative AF in patients undergoing CABG [33]. In our study, serum HSP60, as well as cvHSP, did not discriminate between the stage of AF or AF recurrence. The findings indicate that for baseline serum HSP27, HSP70, cvHSP and HSP60 levels, no clear consensus exists for their use as a biomarker in AF.

\subsection{Limitations and Future Directions: HSP in Relation to Degree of Electropathology}

The current cross-sectional analysis provides information about whether HSP levels in serum samples of AF patients differ from controls in sinus rhythm. To elucidate whether HSP levels predict AF onset, detect early $\mathrm{AF}$ or detect progression of $\mathrm{AF}$, a longitudinal study with repeated blood sampling for HSP measurements and AF testing in subjects with normal sinus rhythm is required. Our control serum HSP27 and HSP70 range is lower than the ranges described in other reports, which can be explained by differences in the analytic assays used $[22,29,30,34]$ and due to differences in disease status of the various patient groups. The absence of difference in serum HSP levels between control and AF patients may be attributed to the clinical nature of the control group.

It is generally accepted that AF is a multifactorial disease and predisposing conditions, e.g., diabetes mellitus, hypertension and higher age were omnipresent in all our study groups, including the control group. It might be possible that the clinical variables we studied were too common and we might need to search for more AF specific parameters, such as markers of structural damage that are found to underlie atrial electrical conduction disorders, i.e., electropathology [35]. It has been suggested that the clinical classification of AF, based on ECG measurements, as presented in the guidelines, is inaccurate for AF staging because it is not related to the degree of atrial electropathology as measured by high-resolution epicardial mapping [36]. Additionally, undiagnosed, silent and/or very short-lasting AF episodes might have been overlooked in control patients and during follow-up of ECV or PVI patients by using ECG measurements. As previous studies revealed exhaustion of HSP levels in atrial tissue of persistent AF patients [18], in future research projects it is recommended to investigate human HSP levels in both serum and atrial tissue and their relationship to parameters related to AF-induced electrical and/or structural remodeling and also structural remodeling-induced (post-operative) AF [37]. Thus, a lack of correlation between HSPs and AF stage in the current study does not negate a role for HSPs as potential biomarker in AF. It is conceivable that serum HSP levels correlate with the degree of electropathology. Future studies with continuous monitoring of electrical parameters and HSP levels should elucidate such an association.

\section{Conclusions}

Serum HSP27, HSP70, cvHSP and HSP60 levels did not differentiate between AF stages and controls in sinus rhythm. Moreover, AF recurrence after ECV or PVI was not associated with baseline HSP levels. However, both HSP27 levels were increased during follow-up in patients with AF recurrence after ablative therapy and may be used as predictors. Future research directed at elucidation of an association between HSP levels and the degree of electropathology is recommended.

Supplementary Materials: The following are available online at http://www.mdpi.com/2073-4409/9/9/2105/s1, Table S1: Concentration of HSPs in serum at baseline of patients without AF and with (paroxysmal, persistent or longstanding persistent) AF, Table S2: Univariate linear regression associations between clinical parameters and HSP levels in baseline serum, Table S3: Multivariate linear regression associations between AF, AF stage and recurrence within one year and HSP levels in baseline serum, corrected for potential confounders, Table S4: Bivariate spearman correlation between clinical parameters and recurrence within 3 months, 6 months and 12 months, Table S5: Clinical characteristics and serum HSP levels for subjects without AF and with (paroxysmal, persistent and longstanding persistent) AF in the ECV group, Table S6: Serum HSP concentrations after one year follow-up, Table S7: Clinical characteristics and serum HSP levels for subjects without AF and with (paroxysmal, persistent and longstanding persistent) AF in the PVI group. 
Author Contributions: Conceptualization, B.J.J.M.B. and N.M.S.d.G.; methodology, B.J.J.M.B., N.M.S.d.G. and E.B.; software, D.M.S.v.M. and E.A.H.L.; formal analysis, D.M.S.v.M., E.A.H.L., K.S.R., L.B.-t.B., M.W. and J.L.; investigation, D.M.S.v.M., E.A.H.L., L.B.-t.B. and A.J.Q.M.M.; resources, D.M.S.v.M., E.A.H.L., L.B.-t.B. and A.J.Q.M.M.; data curation, D.M.S.v.M., E.A.H.L., K.S.R., M.W. and J.L.; writing-original draft preparation, D.M.S.v.M., N.M.S.d.G. and B.J.J.M.B.; writing-review and editing, D.M.S.v.M., E.A.H.L., K.S.R., J.L., M.W., E.B., N.M.S.d.G. and B.J.J.M.B.; visualization, D.M.S.v.M., E.B., N.M.S.d.G. and B.J.J.M.B.; supervision, B.J.J.M.B.; funding acquisition, N.M.S.d.G. and B.J.J.M.B. All authors have read and agreed to the published version of the manuscript.

Funding: This research was funded by the LSH-Impulse grant (2014-40-43100-98-008), Dutch Heart Foundation (2013-2013T144 and 2013-2013T096), CVON-STW2016-14728 AFFIP, NWO-Vidi (2016-91717339 to NMSdG) and Medical Delta (2018).

Acknowledgments: The authors thank all patients who participated in this study; P. Knops, C.P. Teuwen, L.E.J.M. van der Does and E.M.J.P. Mouws for their help with patient inclusion; and M. van Schaik and S. Martens for their help with Western blot and ELISA analyses.

Conflicts of Interest: The authors declare no conflict of interest. The funders had no role in the design of the study; in the collection, analyses, or interpretation of data; in the writing of the manuscript; or in the decision to publish the results.

\section{References}

1. Mozaffarian, D.; Benjamin, E.J.; Go, A.S.; Arnett, D.K.; Blaha, M.J.; Cushman, M.; De Ferranti, S.; Després, J.-P.; Fullerton, H.J.; Howard, V.J.; et al. Executive Summary: Heart Disease and Stroke Statistics-2015 Update: A report from the American Heart Association. Circulation 2015, 131, 434-441. [CrossRef]

2. Heijman, J.; Guichard, J.-B.; Dobrev, D.; Nattel, S. Translational Challenges in Atrial Fibrillation. Circ. Res. 2018, 122, 752-773. [CrossRef] [PubMed]

3. Kirchhof, P.; Benussi, S.; Kotecha, D.; Ahlsson, A.; Atar, D.; Casadei, B.; Castellà, M.; Diener, H.-C.; Heidbuchel, H.; Hendriks, J.; et al. 2016 ESC Guidelines for the management of atrial fibrillation developed in collaboration with EACTS. Europace 2016, 18, 1609-1678. [CrossRef] [PubMed]

4. Turakhia, M.P.; Ullal, A.J.; Hoang, D.D.; Than, C.T.; Miller, J.D.; Friday, K.J.; Perez, M.V.; Freeman, J.V.; Wang, P.J.; Heidenreich, P.A. Feasibility of Extended Ambulatory Electrocardiogram Monitoring to Identify Silent Atrial Fibrillation in High-risk Patients: The Screening Study for Undiagnosed Atrial Fibrillation (STUDY-AF). Clin. Cardiol. 2015, 38, 285-292. [CrossRef] [PubMed]

5. Andrade, J.G.; Deyell, M.W.; Verma, A.; Macle, L.; Champagne, J.; Leong-Sit, P.; Novak, P.; Badra-Verdu, M.; Sapp, J.; Khairy, P.; et al. Association of Atrial Fibrillation Episode Duration with Arrhythmia Recurrence Following Ablation. JAMA Netw. Open 2020, 3, e208748. [CrossRef]

6. January, C.T.; Wann, L.S.; Alpert, J.S.; Calkins, H.; Cigarroa, J.E.; ClevelandJr, J.C.; Conti, J.B.; Ellinor, P.T.; Ezekowitz, M.D.; Field, M.E.; et al. 2014 AHA/ACC/HRS Guideline for the Management of Patients with Atrial Fibrillation. Circulation 2014, 130, e1-e76. [CrossRef]

7. Dudink, E.A.; Weijs, B.; Tull, S.; Luermans, J.G.; Fabritz, L.; Chua, W.; Rienstra, M.; Van Gelder, I.C.; Schotten, U.; Kirchhof, P.; et al. The Biomarkers NT-proBNP and CA-125 are Elevated in Patients with Idiopathic Atrial Fibrillation. J. Atr. Fibrillation 2018, 11, 2058. [CrossRef]

8. Chua, W.; Purmah, Y.; Cardoso, V.R.; Gkoutos, G.; Tull, S.P.; Neculau, G.; Thomas, M.R.; Kotecha, D.; Lip, G.Y.H.; Kirchhof, P.; et al. Data-driven discovery and validation of circulating blood-based biomarkers associated with prevalent atrial fibrillation. Eur. Hear. J. 2019, 40, 1268-1276. [CrossRef]

9. Hui, T.H.; McClelland, R.L.; Allison, M.A.; Rodriguez, C.J.; Kronmal, R.A.; Heckbert, S.R.; Michos, E.D.; Barter, P.J.; Rye, K.-A.; Ong, K.L. The relationship of circulating fibroblast growth factor 21 levels with incident atrial fibrillation: The Multi-Ethnic Study of Atherosclerosis. Atherosclerosis 2018, 269, 86-91. [CrossRef]

10. Zhu, K.; Hung, J.; Divitini, M.; Murray, K.; Lim, E.M.; John, A.S.; Walsh, J.P.; Knuiman, M.W. High-sensitivity cardiac troponin I and risk of incident atrial fibrillation hospitalisation in an Australian community-based cohort: The Busselton health study. Clin. Biochem. 2018, 58, 20-25. [CrossRef]

11. Kubota, Y.; Alonso, Á.; Heckbert, S.R.; Norby, F.L.; Folsom, A.R. Homocysteine and Incident Atrial Fibrillation: The Atherosclerosis Risk in Communities Study and the Multi-Ethnic Study of Atherosclerosis. Hear. Lung Circ. 2019, 28, 615-622. [CrossRef] [PubMed] 
12. Csecsei, P.; Várnai, R.; Nagy, L.; Kéki, S.; Molnár, T.; Illés, Z.; Farkas, N.; Szapáry, L. L-arginine pathway metabolites can discriminate paroxysmal from permanent atrial fibrillation in acute ischemic stroke. Ideggyogy. Szle. 2019, 72, 79-88. [CrossRef] [PubMed]

13. Stanciu, A.E.; Vatasescu, R.-G.; Stanciu, M.M.; Serdarevic, N.; Dorobantu, M. The role of pro-fibrotic biomarkers in paroxysmal and persistent atrial fibrillation. Cytokine 2018, 103, 63-68. [CrossRef]

14. Henning, R.H.; Brundel, B.J.J.M. Proteostasis in cardiac health and disease. Nat. Rev. Cardiol. 2017, 14, 637-653. [CrossRef]

15. Brundel, B.J.J.M.; Shiroshita-Takeshita, A.; Qi, X.; Yeh, Y.-H.; Chartier, D.; Van Gelder, I.C.; Henning, R.H.; Kampinga, H.H.; Nattel, S. Induction of Heat Shock Response Protects the Heart Against Atrial Fibrillation. Circ. Res. 2006, 99, 1394-1402. [CrossRef]

16. Wiersma, M.; Meijering, R.A.M.; Qi, X.; Zhang, D.; Liu, T.; Hoogstra-Berends, F.; Sibon, O.C.M.; Henning, R.H.; Nattel, S.; Brundel, B.J.J.M. Endoplasmic Reticulum Stress Is Associated with Autophagy and Cardiomyocyte Remodeling in Experimental and Human Atrial Fibrillation. J. Am. Hear. Assoc. 2017, 6. [CrossRef]

17. Baler, R.; Dahl, G.; Voellmy, R. Activation of human heat shock genes is accompanied by oligomerization, modification, and rapid translocation of heat shock transcription factor HSF1. Mol. Cell. Boil. 1993, 13, 2486-2496. [CrossRef] [PubMed]

18. Brundel, B.J.J.M.; Henning, R.H.; Ke, L.; Van Gelder, I.C.; Crijns, H.J.; Kampinga, H.H. Heat shock protein upregulation protects against pacing-induced myolysis in HL-1 atrial myocytes and in human atrial fibrillation. J. Mol. Cell. Cardiol. 2006, 41, 555-562. [CrossRef]

19. Kotter, S.; Unger, A.; Hamdani, N.; Lang, P.; Vorgerd, M.; Nagel-Steger, L.; Linke, W.A. Human myocytes are protected from titin aggregation-induced stiffening by small heat shock proteins. J. Cell Biol. 2014, 204, 187-202. [CrossRef]

20. Ghosh, J.G.; Houck, S.A.; Clark, J.I. Interactive Domains in the Molecular Chaperone Human $\alpha$ B Crystallin Modulate Microtubule Assembly and Disassembly. PLoS ONE 2007, 2, e498. [CrossRef]

21. Hu, X.; Li, J.; Van Marion, D.M.; Zhang, D.; Brundel, B.J.J.M. Heat shock protein inducer GGA*-59 reverses contractile and structural remodeling via restoration of the microtubule network in experimental Atrial Fibrillation. J. Mol. Cell. Cardiol. 2019, 134, 86-97. [CrossRef]

22. Hu, Y.-F.; Yeh, H.-I.; Tsao, H.-M.; Tai, C.-T.; Lin, Y.-J.; Chang, S.-L.; Lo, L.-W.; Tuan, T.-C.; Suenari, K.; Li, C.-H.; et al. Electrophysiological Correlation and Prognostic Impact of Heat Shock Protein 27 in Atrial Fibrillation. Circ. Arrhythmia Electrophysiol. 2012, 5, 334-340. [CrossRef] [PubMed]

23. Lanters, E.A.; Van Marion, D.M.S.; Kik, C.; Steen, H.; Bogers, A.J.; Allessie, M.A.; Brundel, B.; De Groot, N.M.S. HALT \& REVERSE: Hsf1 activators lower cardiomyocyt damage; towards a novel approach to REVERSE atrial fibrillation. J. Transl. Med. 2015, 13, 347. [CrossRef]

24. Hijazi, Z.; Aulin, J.; Andersson, U.; Alexander, J.H.; Gersh, B.; Granger, C.B.; Hanna, M.; Horowitz, J.D.; Hylek, E.M.; Lopes, R.D.; et al. Biomarkers of inflammation and risk of cardiovascular events in anticoagulated patients with atrial fibrillation. Heart 2016, 102, 508-517. [CrossRef] [PubMed]

25. Ellinor, P.T.; Low, A.F.; Patton, K.K.; Shea, M.A.; Macrae, C.A. Discordant atrial natriuretic peptide and brain natriuretic peptide levels in lone atrial fibrillation. J. Am. Coll. Cardiol. 2005, 45, 82-86. [CrossRef]

26. Patton, K.K.; Ellinor, P.T.; Heckbert, S.R.; Christenson, R.H.; Defilippi, C.; Gottdiener, J.S.; Kronmal, R.A. N-Terminal Pro-B-Type Natriuretic Peptide Is a Major Predictor of the Development of Atrial Fibrillation. Circulation 2009, 120, 1768-1774. [CrossRef]

27. Takanori, Y.; Yamaguchi, T.; Fukui, A.; Node, K. Bipolar Voltage Mapping for the Evaluation of Atrial Substrate: Can We Overcome the Challenge of Directionality? J. Atr. Fibrillation 2019, 11, 2116. [CrossRef]

28. Kornej, J.; Reinhardt, C.; Kosiuk, J.; Arya, A.; Hindricks, G.; Adams, V.; Husser, D.; Bollmann, A. Response of circulating heat shock protein 70 and anti-heat shock protein 70 antibodies to catheter ablation of atrial fibrillation. J. Transl. Med. 2013, 11, 49. [CrossRef] [PubMed]

29. Mandal, K.; Torsney, E.; Poloniecki, J.; Camm, A.J.; Xu, Q.; Jahangiri, M. Association of High Intracellular, but Not Serum, Heat Shock Protein 70 with Postoperative Atrial Fibrillation. Ann. Thorac. Surg. 2005, 79, 865-871. [CrossRef]

30. Afzal, A.R.; Mandal, K.; Nyamweya, S.; Foteinos, G.; Poloniecki, J.; Camm, A.J.; Jahangiri, M.; Xu, Q. Association of Met439Thr Substitution in Heat Shock Protein 70 Gene with Postoperative Atrial Fibrillation and Serum HSP70 Protein Levels. Cardiology 2008, 110, 45-52. [CrossRef] 
31. Maan, A.; Jorgensen, N.W.; Mansour, M.; Dudley, S., Jr.; Jenny, N.S.; Defilippi, C.; Szklo, M.; Alonso, A.; Refaat, M.M.; Ruskin, J.; et al. Association between Heat Shock Protein-60 and Development of Atrial Fibrillation: Results from the Multi-Ethnic Study of Atherosclerosis (MESA). Pacing Clin. Electrophysiol. 2016, 39, 1373-1378. [CrossRef]

32. Cao, H.; Xue, L.; Xu, X.; Wu, Y.; Zhu, J.; Chen, L.; Chen, D.; Chen, Y. Heat shock proteins in stabilization of spontaneously restored sinus rhythm in permanent atrial fibrillation patients after mitral valve surgery. Cell Stress Chaperones 2011, 16, 517-528. [CrossRef] [PubMed]

33. Oc, M.; Ucar, H.I.; Pinar, A.; Akbulut, B.; Oc, B.; Akinci, S.B.; Akyon, Y.; Kanbak, M.; Boke, E.; Dogan, R. Heat shock protein 60 antibody. A new marker for subsequent atrial fibrillation development. Saudi Med. J. 2007, 28, 844-847. [PubMed]

34. Kardys, I.; Rifai, N.; Meilhac, O.; Michel, J.-B.; Martín-Ventura, J.-L.; Buring, J.; Libby, P.; Ridker, P.M. Plasma Concentration of Heat Shock Protein 27 and Risk of Cardiovascular Disease: A Prospective, Nested Case-Control Study. Clin. Chem. 2008, 54, 139-146. [CrossRef] [PubMed]

35. De Groot, N.M.S.; Houben, R.P.; Smeets, J.L.; Boersma, E.; Schotten, U.; Schalij, M.J.; Crijns, H.; Allessie, M.A. Electropathological Substrate of Longstanding Persistent Atrial Fibrillation in Patients With Structural Heart Disease. Circulation 2010, 122, 1674-1682. [CrossRef]

36. Mouws, E.M.J.P.; Van Der Does, L.J.; Kik, C.; Lanters, E.A.; Teuwen, C.P.; Knops, P.; Bogers, A.J.; De Groot, N.M.S. Impact of the arrhythmogenic potential of long lines of conduction slowing at the pulmonary vein area. Heart Rhythm. 2019, 16, 511-519. [CrossRef]

37. Fakuade, F.E.; Steckmeister, V.; Seibertz, F.; Gronwald, J.; Kestel, S.; Menzel, J.; Pronto, J.R.D.; Taha, K.; Haghighi, F.; Kensah, G.; et al. Altered atrial cytosolic calcium handling contributes to the development of postoperative atrial fibrillation. Cardiovasc. Res. 2020, 162. [CrossRef]

(C) 2020 by the authors. Licensee MDPI, Basel, Switzerland. This article is an open access article distributed under the terms and conditions of the Creative Commons Attribution (CC BY) license (http://creativecommons.org/licenses/by/4.0/). 